SUBJECT AREAS: SEMICONDUCTORS

QUANTUM OPTICS

INORGANIC LEDS

NANOPHOTONICS AND PLASMONICS

Received

10 December 2012

Accepted

20 March 2013

Published

10 April 2013

Correspondence and requests for materials should be addressed to

G.V.A. (astakhov@ physik.uni-wuerzburg. de)

\section{Silicon carbide light-emitting diode as a prospective room temperature source for single photons}

\author{
F. Fuchs', V. A. Soltamov², S. Väth', P. G. Baranov², E. N. Mokhov², G. V. Astakhov' \& V. Dyakonov',3 \\ 'Experimental Physics VI, Julius-Maximilian University of Würzburg, 97074 Würzburg, Germany, ${ }^{2}$ loffe Physical-Technical Institute, \\ 194021 St. Petersburg, Russia, ${ }^{3}$ Bavarian Center for Applied Energy Research (ZAE Bayern), 97074 Würzburg, Germany.
}

Generation of single photons has been demonstrated in several systems. However, none of them satisfies all the conditions, e.g. room temperature functionality, telecom wavelength operation, high efficiency, as required for practical applications. Here, we report the fabrication of light-emitting diodes (LEDs) based on intrinsic defects in silicon carbide $(\mathrm{SiC})$. To fabricate our devices we used a standard semiconductor manufacturing technology in combination with high-energy electron irradiation. The room temperature electroluminescence (EL) of our LEDs reveals two strong emission bands in the visible and near infrared (NIR) spectral ranges, associated with two different intrinsic defects. As these defects can potentially be generated at a low or even single defect level, our approach can be used to realize electrically driven single photon source for quantum telecommunication and information processing.

obust and cheap light sources emitting single photons on demand are at the heart of many demanding optical technologies ${ }^{1,2}$. Single photon emission has been demonstrated in a variety of systems, including atoms $^{3}$, ions $s^{4}$, molecules ${ }^{5-7}$, quantum dots $(\mathrm{QDs})^{8,9}$ and color centers in diamond ${ }^{10,11}$. The most significant progress has been achieved for $\mathrm{QDs} \mathrm{s}^{12-14}$, however, the necessity to use cryogenic temperatures and high inhomogeneity (the emission wavelength is individual for each QD) make this system impractical. Electrically driven single photon sources in the visible spectral range have also been demonstrated using nitrogen-vacancy (NV) centers in diamond ${ }^{15,16}$, but the compatibility of this system with the present-day integrated circuits manufacturing is not obvious.

The operation principle of single photon sources is based on the quantum mechanical properties of a single two-level system. When a single photon is desired, this system is put into the excited state by an external stimulus, and a single photon is emitted upon relaxation into the ground state. A perspective approach to fabricate an efficient, room temperature single photon source based on this principle is to use color centers in semiconductors. In our work, we exploit two defect centers in $\mathrm{SiC}$, the so-called $\mathrm{D}_{1}$ defect ${ }^{17}$ and the silicon vacancy $\left(\mathrm{V}_{\mathrm{Si}}\right)$ defect ${ }^{18}$, making two-color LED [Fig. 1(a)].

Remarkably, the $\mathrm{V}_{\mathrm{Si}}$ defects in $\mathrm{SiC}$ comprise the technological advantages of semiconductor quantum dots and the unique quantum properties of the $\mathrm{NV}$ defects in diamond ${ }^{19}$. In particular, $\mathrm{V}_{\mathrm{Si}}$ spin qubits can be optically initialized and read out ${ }^{18,19}$, and, therefore, our demonstration of room temperature EL from $\mathrm{V}_{\mathrm{Si}}$ defects is an important step towards realization of all-electrical control of $\mathrm{V}_{\mathrm{Si}}$ spins. Further, the $\mathrm{V}_{\mathrm{Si}}$ EL reveals a broad-band emission spectrum in NIR $(850-1050 \mathrm{~nm})$, where the absorption of silica glass optical fibers is relatively weak. While this spectrum range is still below the telecom window $(1.3 \mu \mathrm{m})$, it can be changed in the direction of longer wavelengths by proper choosing over family of deep defect centers in different $\mathrm{SiC}$ polytypes ${ }^{20}$. Alternatively, the frequency conversion of NIR photons to a telecom wavelength can be applied ${ }^{21,22}$. Therefore, the integration of defect-based $\mathrm{SiC}$ LEDs with existing telecommunication infrastructure seems feasible.

$\mathrm{SiC}$ with highly developed device technologies (e.g. MOSFETS, MEMS, sensors) is a very attractive material for practical applications. SiC is also known as the material on which the first LED has been created $^{23}$. Until the 90's, SiC was used for commercial yellow and blue LEDs, but later it was replaced with GaN. One of the disadvantages of $\mathrm{SiC}$ for opto-electronics is its indirect bandgap. As a consequence, direct band-to-band (BB) radiative recombination is inefficient, compared to recombination via subgap states [Fig. 1(b)]. Engineering and isolating single defects with proper transition energy on demand can open a route for an efficient electrical single photons source. 

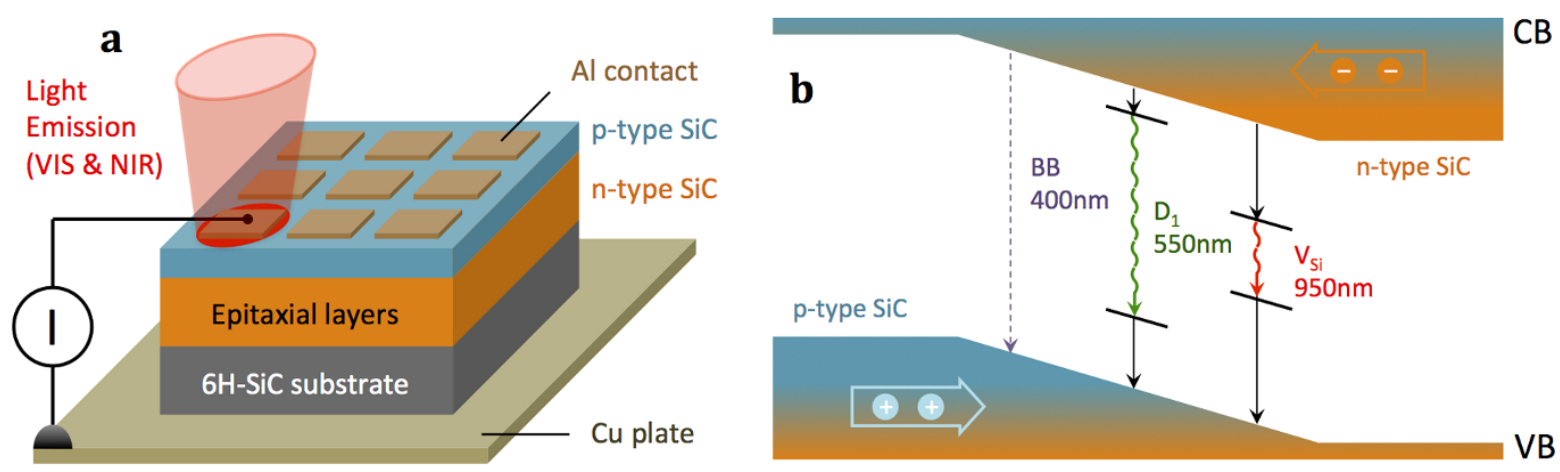

Figure $1 \mid$ SiC LED with intrinsic defects. (a) A scheme of the SiC LED. (b) Electron-hole recombination through the $\mathrm{D}_{1}$ and $\mathrm{V}_{\mathrm{Si}}$ defects results in the $550 \mathrm{~nm}$ and $950 \mathrm{~nm}$ emission bands, respectively. The radiative band-to-band recombination (BB) at $400 \mathrm{~nm}$ is inefficient because SiC is an indirect bandgap semiconductor.

\section{Results}

A scheme of the SiC LED structure, consisting of a single p-n junction, is presented in Fig. 1(a). Intrinsic defects in these structures were generated by electron irradiation. We mount LED samples on a $\mathrm{Cu}$ plate serving as the back electrode. Upon applying voltage between one of the $\mathrm{Al}$ contacts and the $\mathrm{Cu}$ plate the luminescence glow is seen by the naked eye [Fig. 2(a)]. The room temperature EL spectrum of one of our LEDs is presented in Fig. 2(b). It consists of two broad emission bands, labeled as $\mathrm{D}_{1}$ and $\mathrm{V}_{\mathrm{Si}}$. The corresponding recombination processes at the $\mathrm{p}-\mathrm{n}$ junction are schematically shown in Fig. 1(b).

We now discuss the EL bands of Fig. 2(b) in details. The emission energies are seen to be significantly smaller than the bandgap of $6 \mathrm{H}$ $\mathrm{SiC}(3.05 \mathrm{eV})$. We, therefore, ascribe them to the defects in SiC. The emission in the spectral range $450-650 \mathrm{~nm}$ is characteristic of the $\mathrm{D}_{1}$ zero-phonon lines (ZPLs) and their phonon replicas ${ }^{24}$, merging together at room temperature. The nature of this defect is still not clear - several models have been proposed, including a boundexciton-like center ${ }^{24}$ and a first-neighbor antisite pair $\mathrm{Si}_{\mathrm{C}}-\mathrm{C}_{\mathrm{Si}^{2}}{ }^{25}$. The second emission band in the NIR spectral range 850 $1050 \mathrm{~nm}$ coincides with the photoluminescence (PL) spectrum of the silicon vacancy defects $\mathrm{V}_{\mathrm{Si}}{ }^{26}$ in the reference $6 \mathrm{H}-\mathrm{SiC}$ bulk sample.

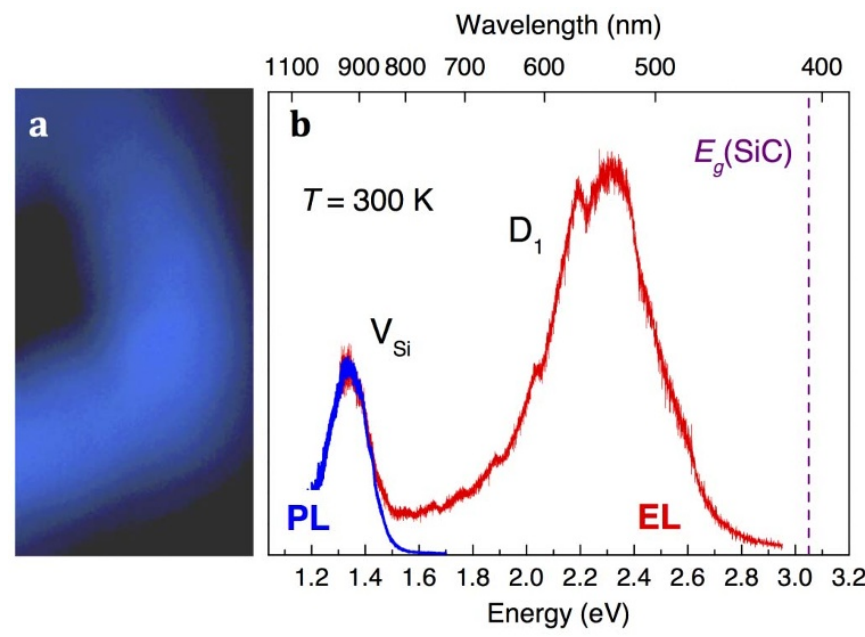

Figure $2 \mid$ Room-temperature electroluminescence of intrinsic defects in SiC. (a) An image of the luminous LED around an Al contact. (b) Electroluminescence (EL) spectrum of the SiC LED and photoluminescence $(\mathrm{PL})$ spectrum of the reference $\mathrm{SiC}$ sample recorded at room temperature. The PL spectrum is excited by a He-Ne laser with $E_{\text {exc }}=$ $1.96 \mathrm{eV}(633 \mathrm{~nm})$. The bandgap of $6 \mathrm{H}-\mathrm{SiC}$ is $E_{g}(\mathrm{SiC})=3.05 \mathrm{eV}$.
To prove this interpretation we repeat the experiment of Fig. 2(b) at a temperature of $77 \mathrm{~K}$ [see Fig. 3(a)], when the spectroscopic features, individual for each defect, can be resolved. The results are summarized in Fig. 3 and below we discuss them in detail.

\section{Discussion}

First, we demonstrate the presence of $\mathrm{V}_{\mathrm{Si}}$ defects in our LED structures. Figure 3(b) shows photoluminescence (PL) spectrum recorded under excitation with the energy $E_{\text {exc }}=1.96 \mathrm{eV}$, which is below the $\mathrm{D}_{1}$ emission energy. Three ZPLs at $1.368 \mathrm{eV}, 1.398 \mathrm{eV}$ and $1.434 \mathrm{eV}$ are the well known fingerprint of the $\mathrm{V}_{\mathrm{Si}}$ defects in $6 \mathrm{H}-\mathrm{SiC}^{27}$. These three ZPLs originate from three nonequivalent crystallographic sites


Figure $3 \mid \mathrm{EL}$ and PL spectra of SiC LED recorded at $77 \mathrm{~K}$.

(a) Comparison of the EL (shaded area) and PL spectra under excitation with an energy $E_{\text {exc }}=2.62 \mathrm{eV}(473 \mathrm{~nm})$. Inset: The same, but shown in the spectral range where the strongest $\mathrm{V}_{\mathrm{Si}} \mathrm{ZPL}$ (V1) is expected. (b) PL spectrum obtained under excitation with a He-Ne laser with $E_{\text {exc }}=1.96 \mathrm{eV}$ $(633 \mathrm{~nm})$. The V1, V2 and V3 ZPLs characteristic for the $\mathrm{V}_{\mathrm{Si}}$ defects in SiC are clearly seen. (c) Integral intensity of the $\mathrm{V}_{\mathrm{Si}}$ and $\mathrm{D}_{1}$ emission bands [the shaded areas in (a)] as a function of LED current. The solid line is a fit (see text for details). 
in this $\mathrm{SiC}$ polytype and are frequently labeled as V3, V2, and V1, respectively. The highest $\mathrm{ZPL}$ intensity is observed for $\mathrm{V}_{\mathrm{Si}}(\mathrm{V} 1)$.

Second, we demonstrate that the $\mathrm{V}_{\mathrm{Si}}(\mathrm{V} 1)$ defect can be electrically driven. Figure 3 (a) shows EL spectrum recorded at $T=77 \mathrm{~K}$. In contrast to room temperature [Fig. 2(b)], the $\mathrm{D}_{1}$ emission dominates in the spectrum. The reason is the much higher concentration of $D_{1}$ defects than of $\mathrm{V}_{\mathrm{Si}}$ defects. However, at room temperature most of the $D_{1}$ defects are ionized due to the thermal activation of the defectbound electrons in the conduction band. This is consistent with a small activation energy (about $60 \mathrm{meV}^{28,29}$ ) of the $\mathrm{D}_{1}$ defect in a different polytype $4 \mathrm{H}$. With lowering temperature, the activation process becomes inefficient and EL (PL) intensity increases. On the other hand, the activation energy of the $\mathrm{V}_{\mathrm{Si}}$ defects is much larger and their intensity weakly depends on temperature. Indeed, we observe the characteristic $\mathrm{V}_{\mathrm{Si}}(\mathrm{V} 1) \mathrm{ZPL}$ at $1.434 \mathrm{eV}$ in the $\mathrm{EL}$ spectrum [the inset of Fig. 3(a)]. The larger ZPL spectral width in EL compared to PL is most probably caused by current-induced charge fluctuations in the vicinity of $\mathrm{V}_{\mathrm{Si}}$ defects, leading to an increase of inhomogeneous broadening.

Third, we verify that the electrical excitation of the $\mathrm{V}_{\mathrm{Si}}(\mathrm{V} 1)$ defect shown in the inset of Fig. 3(a) is not due to the re-emission process via $\mathrm{D}_{1}$. We excite into the maximum of the $\mathrm{D}_{1}$ band $(2.62 \mathrm{eV})$, leading to the Stokes shift of the $\mathrm{D}_{1}$ emission spectrum [Fig. 3(a)]. The laser intensity per area is several orders of magnitude higher than that of the $\mathrm{D}_{1}$ emission, but no significant enhancement of the $\mathrm{V}_{\mathrm{Si}}(\mathrm{V} 1) \mathrm{PL}$ is observed. This means that while the reemission may potentially take place, it is inefficient as compared to electrical excitation. Therefore, we conclude, the recombination of electrically injected electrons and holes is responsible for the $\mathrm{V}_{\mathrm{Si}}$ EL, as schematically shown in Fig. 1(b).

Finally, we present an input-output characteristic of one of our LED devices [Fig. 3(c)]. A clear tendency to saturation of the emission intensity $P$ with injection current $I$ is seen. This behavior can be reasonably well described by the equation $P \propto I /\left(I+I_{0}\right)$, corresponding to the simple model when the carrier capturing rate by the defects is proportional to the injection current. From this fit we estimate the characteristic saturation current $I_{0}=10 \mathrm{~mA}$. It is higher than that in QD-based single photon LEDs ${ }^{30}$ and comparable to that in NV-based single photon LEDs ${ }^{16}$.

In conclusion, we generated intrinsic defects in $\mathrm{SiC}$ devices and demonstrated that these defects can be electrically driven, resulting in the efficient EL with emission energies well below the SiC bandgap. Our LEDs are two-color in a sense that they show two spectrally different emission bands associated with different defects. The $\mathrm{D}_{1}$ defects show EL in visible, which is intense at low temperatures but quenches with rising temperature. The $\mathrm{V}_{\mathrm{Si}}$ defects emit in NIR even at room temperature. By varying the irradiation dose one can control defect concentration, which should allow to isolate single defects, similar to single NV centers in diamond or single semiconductor QDs. Because isolated defects are ideal single photon emitters, our findings open a new way to fabricate cheap and robust LEDs emitting single photons on demand.

\section{Methods}

The LED structures used in our experiments were grown on a n-type $6 \mathrm{H}$-polytype $\mathrm{SiC}$ substrate. First, an epitaxial $15-\mu \mathrm{m}$-thick $\mathrm{SiC}$ layer was grown by the sublimation method. It is n-type and contains $\mathrm{N}\left(3 \times 10^{18} \mathrm{~cm}^{-3}\right)$ and $\mathrm{Ga}\left(2 \times 10^{18} \mathrm{~cm}^{-3}\right)$. The layer is followed by a p-type $\mathrm{SiC}$ layer of thickness $5 \mu \mathrm{m}$ grown at a temperature of $2300^{\circ} \mathrm{C}$ in $\mathrm{Ar}$ atmosphere in the presence of $\mathrm{Al}$ vapors (pressure $100 \mathrm{~Pa}$ ). This results in the concentration of $\mathrm{Al}$ acceptors of $\mathrm{ca} .10^{20} \mathrm{~cm}^{-3}$. In order to generate intrinsic defects at the p-n junction the samples were irradiated with $0.9 \mathrm{MeV}$ electrons to a dose of $10^{18} \mathrm{~cm}^{-2}$. After irradiation, the samples were annealed for 1 minute in $\mathrm{Ar}$ atmosphere at a temperature of $1700^{\circ} \mathrm{C}$. At the final stage, $0.4 \times 0.4 \mathrm{~mm}^{2} \mathrm{Al}$ contacts were deposited on the top of the p-type SiC layer.

EL and PL were recorded using LabRAM system for microscopy (Horiba Scientific) equipped with a CCD camera. In low temperature experiments, the samples were inserted in liquid nitrogen and the $\mathrm{Cu}$ back electrode plate was also used as a cold finger.

1. Santori, C., Fattal, D. \& Yamamoto, Y. Single-photon devices and applications. Wiley-VCH, Weinheim, 2010.
2. Eisaman, M. D., Fan, J., Migdall, A. \& Polyakov, S. V. Invited Review Article: Single-photon sources and detectors. Rev. Sci. Instr. 82, 071101 (2011).

3. McKeever, J. et al. Deterministic generation of single photons from one atom trapped in a cavity. Science 303, 1992-1994 (2004).

4. Keller, M., Lange, B., Hayasaka, K., Lange, W. \& Walther, H. Continuous generation of single photons with controlled waveform in an ion-trap cavity system. Nature 431, 1075-1078 (2004).

5. Brunel, C., Lounis, B., Tamarat, P. \& Orrit, M. Triggered source of single photons based on controlled single molecule fluorescence. Phys. Rev. Lett. 83, 2722-2725 (1999).

6. Lounis, B. \& Moerner, W. E. Single photons on demand from a single molecule at room temperature. Nature 407, 491-493 (2000).

7. Nothaft, M. et al. Electrically driven photon antibunching from a single molecule at room temperature. Nat. Commun. 3, 628 (2012).

8. Michler, P. et al. A quantum dot single-photon turnstile device. Science $\mathbf{2 9 0}$, 2282-2285 (2000).

9. Yuan, Z. et al. Electrically driven single-photon source. Science 295, 102-105 (2002).

10. Kurtsiefer, C., Mayer, S., Zarda, P. \& Weinfurter, H. Stable solid-state source of single photons. Phys. Rev. Lett. 85, 290-293 (2000).

11. Neu, E. et al. Single photon emission from silicon-vacancy colour centres in chemical vapour deposition nano-diamonds on iridium. New J. Phys. 13, 025012 (2011).

12. Pelton, M. et al. Efficient source of single photons: A single quantum dot in a micropost microcavity. Phys. Rev. Lett. 89, 233602 (2002).

13. Muller, A., Fang, W., Lawall, J. \& Solomon, G. S. Creating polarization-entangled photon pairs from a semiconductor quantum dot using the optical stark effect. Phys. Rev. Lett. 103, 217402 (2009).

14. Heindel, T. et al. Quantum key distribution using quantum dot single-photon emitting diodes in the red and near infrared spectral range. New J. Phys. 14, 083001 (2012).

15. Lohrmann, A. et al. Diamond based light-emitting diode for visible single-photon emission at room temperature. Appl. Phys. Lett. 99, 251106 (2011).

16. Mizuochi, N. et al. Electrically driven single-photon source at room temperature in diamond. Nat. Photon. 6, 299-303 (2012).

17. Patrick, L. \& Choyke, W. J. Photoluminescence of radiation defects in ionimplanted 6H SiC. Phys. Rev. B 5, 3253-3259 (1972).

18. Baranov, P. G. et al. Silicon vacancy in $\mathrm{SiC}$ as a promising quantum system for single-defect and single-photon spectroscopy. Phys. Rev. B 83, 125203 (2011).

19. Riedel, D. et al. Resonant addressing and manipulation of silicon vacancy qubits in silicon carbide. Phys. Rev. Lett. 109, 226402 (2012).

20. Magnusson, B. \& Janzén, E. Optical characterization of deep level defects in SiC Mater. Sci. Forum 483-485, 341-346 (2005).

21. Zaske, S. et al. Visible-to-telecom quantum frequency conversion of light from a single quantum emitter. Phys. Rev. Lett. 109, 147404 (2012).

22. Greve, K. D. et al. Quantum-dot spin-photon entanglement via frequency downconversion to telecom wavelength. Nature 491, 421-425 (2012).

23. Zheludev, N. The life and times of the LED - a 100-year history. Nat. Photon. 1, 189-192 (2007)

24. Fissel, A., Richter, W., Furthmüller, J. \& Bechstedt, F. On the nature of the $\mathrm{D}_{1}$-defect center in SiC: A photoluminescence study of layers grown by solid-source molecular-beam epitaxy. Appl. Phys. Lett. 78, 2512-2514 (2001).

25. Gali, A. et al. Correlation between the antisite pair and the $\mathrm{D}_{\mathrm{I}}$ center in SiC. Phys. Rev. B 67, 155203 (2003).

26. Vainer, V. S. \& Ilyin, V. A. Electron spin resonance of exchange-coupled vacancy pairs in hexagonal silicon carbide. Sov. Phys. Solid State 23, 2126-2133 (1981).

27. Wagner, M. T. et al Electronic structure of the neutral silicon vacancy in $4 \mathrm{H}$ and 6H SiC. Phys. Rev. B 62, 16555-16560 (2000).

28. Egilsson, T., Ivanov, I. G., Nenry, A. \& Janzén, E. Pseudo-donors in SiC. Mater. Sci. Forum 338-342, 647-650 (2000).

29. Storasta, L. et al. Pseudodonor nature of the $\mathrm{D}_{\mathrm{I}}$ defect in $4 \mathrm{H}-\mathrm{SiC}$. Appl. Phys. Lett. 78, 46-48 (2001).

30. Böckler, C. et al. Electrically driven high-Q quantum dot-micropillar cavities. Appl. Phys. Lett. 92, 091107 (2008).

\section{Acknowledgments}

We acknowledge financial support by the Bavarian Ministry of Economic Affairs, Infrastructure, Transport and Technology as well as by the Ministry of Education and Science, Russia, under the Contracts No. 8017 and No. 8568, the Programs of the Russian Academy of Sciences and by the RFBR. V.A.S. acknowledges partial support by the grant of the President of the Russian Federation No. 14.122.13.6053-MK. This publication was funded by the German Research Foundation (DFG) and the University of Würzburg in the funding programme Open Access Publishing.

\section{Author contributions}

F.F. conducted the experiments and analyzed the experimental data; V.A.S. and S.V. conducted the experiments; E.N.M. fabricated the samples; P.G.B., G.V.A. and V.D. conceived the experiments; G.V.A. wrote the main manuscript text; F.F. and V.D. critically reviewed and corrected the manuscript; all authors discussed the results. 


\section{Additional information}

Competing financial interests: The authors declare no competing financial interests. License: This work is licensed under a Creative Commons

Attribution-NonCommercial-NoDerivs 3.0 Unported License. To view a copy of this license, visit http://creativecommons.org/licenses/by-nc-nd/3.0/
How to cite this article: Fuchs, F. et al. Silicon carbide light-emitting diode as a prospective room temperature source for single photons. Sci. Rep. 3, 1637; DOI:10.1038/srep01637 (2013). 